\title{
La dirección integrada de proyectos aplicada a los proyectos comunitarios.
}

\section{The integrated project management applied to community projects.}

Medinilla Sarduy Alexis. ${ }^{1}$, Efraín Velasteguí López. ${ }^{2}$, José A. Bran Cevallos. ${ }^{3}$ María Angelica Gaibor Becerra., ${ }^{4}$ \& Mireya Katerine Pazmiño Arregui. ${ }^{5}$

Recibido: 13-03-2017 / Revisado: 07-05-2017 Aceptado: 05-06-2018/ Publicado: 01-07-2018

\begin{abstract}
.
DOI: $\underline{\text { https://doi.org/10.33262/cienciadigital.v2i3.144 }}$
\end{abstract}

Tourism over time, has become one of the main drivers of the world economy. With this development it has become evident and necessary to pay much more attention to cultural and social aspects because they are very demanded elements by contemporary tourism.

Integrated Project Management has been developed around the world for the last 25 years. However, today, it has matured and has given rise to a set of techniques and disciplines that, when properly used, lead to the achievement of the purpose of a project.

The present study constitutes a qualitative investigation, that arose from the necessity of the Council of the Provincial Administration of Cienfuegos to insert the area object of study to the development of the Historical Center of the city of Cienfuegos, and that is why it has as general objective: Design the bases for a community project (tourist-cultural) in the Historical Center of the city of Cienfuegos. Using the designed instrument, after demonstrating its reliability and validity, the area under study is characterized and the adaptability of the area to tourism development is analyzed. The phases and activities exposed in response to the research problem are designed using the proposed procedure for Community Projects.

${ }^{1}$ Universidad de La Habana, Facultad de Turismo, La Habana, Cuba, alexlester.medy@ gmail.com

${ }^{2}$ Universidad Técnica de Cotopaxi, Ext. La Maná, Cotopaxi, Ecuador, luis.velastegui7838@utc.edu.ec

${ }^{3}$ Universidad de Guayaquil, Facultad de Ing. Industrial, Guayaquil, Ecuador, jose.branc@ug.edu.ec

${ }^{4}$ Universidad Estatal de Bolívar, Bolívar, Ecuador, aleviga03@yahoo.es

${ }^{5}$ Universidad Estatal de Bolívar, Fac. de Jurisprudencia, Bolívar, Ecuador, mireyapazarregui@yahoo.com 
Keywords: Integrated Project Management, Tourism, Community Project, Reliability and Validity.

\section{Resumen.}

El turismo a lo largo del tiempo, se ha convertido en uno de los principales dinamizadores de la economía mundial. Con este desarrollo se ha hecho evidente y necesario prestarle mucha más atención a los aspectos culturales y sociales por ser elementos muy demandados por el turismo contemporáneo.

La Gestión Integrada de Proyecto se ha desarrollado en el mundo durante los últimos 25 años. Sin embargo, hoy, ha madurado y ha dado lugar a un conjunto de técnicas y disciplinas que, cuando se utilizan adecuadamente, conducen a la obtención de la finalidad de un proyecto.

El presente estudio constituye una investigación cualitativa, que surgió a partir de la necesidad del Consejo de la Administración Provincial de Cienfuegos de insertar el área objeto de estudio al desarrollo del Centro Histórico de la ciudad de Cienfuegos, $y$ es por ello que la misma tiene como objetivo general: Diseñar las bases para un proyecto comunitario (turístico-cultural) en el Centro Histórico de la ciudad de Cienfuegos.

Haciendo uso del instrumento diseñado, después de demostrar su fiabilidad y validez, se caracteriza la zona objeto de estudio y se analiza la adaptabilidad de la zona al desarrollo turístico. Las fases y actividades expuestas como respuesta al problema de investigación se diseñan haciendo uso del procedimiento propuesto para los Proyectos Comunitarios.

Palabras Claves: Gestión Integrada de Proyecto, Turismo, Proyecto Comunitario, Fiabilidad y Validez.

\section{Introducción.}

En la medida en que los mercados fructifican y se vuelven más competitivos, la retención del cliente cobra una importancia estratégica: es sabido que cuesta varias veces más obtener un cliente nuevo que volver a venderle a un cliente actual.

Por otra parte, el impacto negativo que una mala experiencia no resuelta tiene sobre las recomendaciones, y su efecto sobre la imagen de un destino o sus productos y servicios, están ampliamente demostrados. Las consecuencias que un servicio aparente o incompleto tienen sobre las percepciones de un cliente son nefastas: deterioran la confianza y destruyen 
el vínculo. Por el contrario, una atención rápida, idónea y sin errores funciona como el fidelizador más eficaz que se pueda desarrollar.

Es por ello que las herramientas estratégicas para la organización y planificación de los servicios constituyen un arsenal de sumo valor para la proliferación y sustento de las empresas en el mercado.

Según Zamora (2009), en la década del 60-70 del siglo XX, se comienza a aplicar limitadamente una disciplina llamada Dirección de Proyectos en los Estados Unidos en sectores relacionados con proyectos únicos muy complejos entre ellos, los aeroespaciales acometidos por The National Aeronautics and Space Administration (NASA), centrales nucleares etc. Seguidamente se generalizan a la construcción de edificios, a proyectos de investigación y desarrollo, de telecomunicación e informática, su uso se extendió hasta los proyectos sociales y políticos.

Dada la necesidad de proceder con carácter multidisciplinar logrando integrarla con demás disciplinas y respondiendo a la necesidad de su aplicación en los cambios turbulentos en el mundo empresarial con el devenir de los años se comienza a denominar como Dirección Integrada de Proyecto (DIP).

Según Fajardo y Manresa (2012), en Cuba su aplicación al turismo se inicia bajo la asesoría del Ing. Ángel Gómez Trueba y del profesor Rafael de Heredia, uno de los principales representantes en el mundo europeo de la disciplina. Según los especialistas de la Dirección de Desarrollo del Ministerio de Turismo en la actualidad se están llevando a cabo numerosos proyectos encaminados al desarrollo turístico de zonas con potencialidades, lo cual es coherente con la política económico-social que fomenta el desarrollo local a partir de proyectos de inversión. Aunque también reconocen que en las entidades que brindan servicios asociados a la hospitalidad no se implementa la DIP para crear nuevos productosservicios o mejorarlos, lo que constituye una debilidad pues los proyectos se insertan en la planificación estratégica de las empresas turísticas y son un instrumento que les permite adaptarse a las condiciones cambiantes del entorno y a las nuevas tendencias de la demanda turística internacional, incrementando así su posición competitiva.

La industria de los cruceros actualmente está en aumento y en consecuencia de los retos a la gestión y al desarrollo de esta modalidad turística. Este tema se ha retomado en Cuba a raíz del desarrollo del modelo económico como una oportunidad que tiene este destino caribeño para potenciar sus valiosos tesoros culturales e impulsar el desarrollo turístico en diferentes localidades, en función de obtener mayores ingresos para la economía.

La ciudad de Cienfuegos posee numerosas condiciones a partir de su preciosa bahía, que la convierten en un destino de cruceros. Ciudad emblemática, fundada por franceses, con un centro histórico que ostenta la categoría Patrimonio de la Humanidad. Es considerada una 
zona turística que cuenta con una diversidad de atractivos como son: museos, hermosos parques, sitios históricos y artísticos, hoteles y espacios enriquecidos por la auténtica cultura cienfueguera. Para dar respuesta al arribo de cruceros a la ciudad, se retoma un muelle que anteriormente tenía funciones comerciales respondiendo en parte a la empresa de astilleros Astisur y el resto a la Empresa de Terminales Mambisas de Cienfuegos para la manipulación de carga general, fundamentalmente dieta básica.

Este muelle está situado en Ave 46 e/ 23 y litoral a 300m del centro la ciudad, este recorrido está comprendido entre las calles 19 (nombrada Arango) y 37 (nombrada Calzada de la Independencia) y más conocida como Prado. Siendo, para los cruceristas, la primera impresión e imagen de la zona turística ciudad de Cienfuegos. Considerada esta zona, el objeto de estudio para la presente investigación.

Esta área se encuentra mayoritariamente en el Consejo Popular Centro Histórico, y está caracterizada por una agresiva situación social, por la ausencia de atractivos y por el desaprovechamiento de los valores históricos y culturales de la localidad, convirtiéndose en el área menos desarrollada del Centro Histórico de Cienfuegos. Si se añade a este hecho que es una zona depauperada estéticamente con un alto grado de marginalidad, se crea una situación problémica que amerita una investigación científica que aporte a su solución.

Es por ello que se define como Problema de Investigación: ¿Cómo aprovechar los valores históricos y culturales de la localidad para la inserción en el Centro Histórico de la ciudad mejorando la situación de la comunidad?

Para esto se define como Objetivo General: Diseñar las bases para un proyecto comunitario (turístico-cultural) en el Centro Histórico de la ciudad de Cienfuegos.

\section{Desarrollo.}

\section{Marco Teórico Referencial.}

La Gestión Integrada de Proyecto (GIP) se ha desarrollado en el mundo durante los últimos 25 años. Hasta el año 1970 el concepto y el término eran prácticamente desconocidos; hoy, por el contrario, la GIP ha madurado y ha dado lugar a un conjunto de técnicas y disciplinas que, cuando se utilizan adecuadamente, conducen a la obtención de la finalidad de un proyecto.

Para definir el sistema "Proyecto" se toma de David I. Cleland y William R. Kling en su obra "Systems Analysis and Project Management" por ser la que mejor se adecua a este caso. Según estos autores, proyecto es "la combinación de recursos humanos y no humanos reunidos en una organización temporal para conseguir un propósito determinado". En esta definición hay algunos elementos que son básicos para la comprensión de lo que es un Proyecto. 
En primer lugar, se trata de una combinación de recursos humanos y no humanos; esto indica que se trata de, bajo un solo equipo o grupo, utilizar personas que han de manejar otros recursos, entre ellos los económicos y los de tiempo, así como los de carácter tecnológico y a veces los ecológicos. Todos estos recursos han de estar reunidos en una organización temporal, o sea, que tiene un principio y un fin en el tiempo; es muy importante resaltar esta característica, ya que si estos recursos antes mencionados se reunieran en una organización que tendiera a perpetuarse estaríamos en el caso de una Empresa y no de un Proyecto.

Por ello, es el carácter de temporalidad lo que define con mayor claridad el Proyecto. Estos recursos que han de estar reunidos en una organización temporal, han de conseguir un propósito determinado. Se hace notar que la obtención de un propósito determinado - que es el objeto del Proyecto -, habrá de encajarse dentro de los fines generales de la Empresa o Institución que realiza el Proyecto.

Otra definición de "Proyecto" es la dada por el Project Management Institute de los E.U.A, en su "Project Management Book of Knowledge" (PMBOK). Esta es que "Proyecto es cualquier realización con punto de comienzo definido mediante los que se identifican, entre otras cosas, la fecha de su terminación...”.

En la práctica, la mayoría de los Proyectos dependen de recursos finitos y limitados con los que hay que cumplir los objetivos. En general, los Proyectos nacerán como respuesta a la necesidad de cumplimiento de determinados objetivos de cualquier Empresa o Institución y estarán enmarcados dentro de la finalidad de ésta.

El enfoque de optimización se centra en la eficacia que supone aprovechar las oportunidades para crear resultados, incluso cambiando las condiciones existentes. Es conveniente tener claras las ideas sobre eficiencia y eficacia. Eficiencia significa hacer mejor lo que ya se está haciendo, está relacionada con hacer las cosas bien y es un requisito mínimo para alcanzar el éxito, mientras que la eficacia es la base del éxito y está relacionada con hacer lo que se debe hacer.

De las definiciones dadas anteriormente, fácilmente se deduce lo que es y en lo que consiste la Gestión Integrada de Proyecto. Según Heredia (1995), puede decirse que es "el Management aplicado a un Proyecto", o bien, siendo más explícito, definirlo como "el proceso de optimización de los recursos puestos a disposición del Proyecto, con el fin de obtener sus objetivos"; también puede definirse por "el proceso de conducción del esfuerzo organizativo, en el sentido del liderazgo para obtener los objetivos del Proyecto". 


\section{Marco Metodológico.}

La metodología que se sigue en este trabajo es cualitativa, puesto que la misma se fundamenta en un proceso inductivo- deductivo, donde se analiza, se interpreta y se describe informaciones o realidades de hechos en su entorno natural, para luego generar perspectivas teóricas en cuanto al fenómeno analizado y lograr una correcta interpretación del mismo.

Para la realización de la presente investigación se siguió la siguiente trayectoria metodológica:

Figura No. 1: Trayectoria metodológica.

\begin{tabular}{|l|l|}
\hline $1-$ & $\begin{array}{l}\text { Argumentación del marco referencial en donde se analizaron los aspectos } \\
\text { teóricos-metodológicos que sustentan el estudio en sí. }\end{array}$ \\
\hline 2- & $\begin{array}{l}\text { Caracterización de la ciudad de Cienfuegos como destino turístico, así como } \\
\text { de su Centro Histórico. }\end{array}$ \\
\hline $3-$ & - Diagnóstico de la situación real existente en la zona objeto de estudio. \\
\hline 4- & - Justificación y antecedentes del proyecto a realizar. \\
\hline $5-$ & - Creación del procedimiento para proyectos comunitarios. \\
\hline 6- & Presentación de la propuesta de proyecto. Diseño operativo. \\
\hline
\end{tabular}

Fuente: Elaboración propia.

A continuación, se mencionan los métodos utilizados en la trayectoria metodológica antes mencionada:

Del nivel teórico:

- Histórico-lógico

- Analítico-sintético

Hipotético-deductivo 


\section{Del nivel empírico:}

- Revisión bibliográfica y documental

\section{- Observación Científica}

Encuesta: Los factores que se tienen en cuenta para el diseño del instrumento son: condiciones físicas, condiciones ambientales, servicios públicos, servicios privados, seguridad ciudadana y adaptabilidad de la zona al desarrollo turístico.

\section{Métodos estadísticos-matemáticos}

Para comprobar la fiabilidad y la validez del instrumento que se diseña se aplican una serie de pruebas estadísticas como son: el análisis de la fiabilidad mediante el coeficiente del Alpha de Cronbach, y la validez de contenido, de criterio y de constructo con sus correspondientes coeficientes y correlaciones.

- Análisis de fiabilidad: Coeficiente Alfa de Cronbach.

- Análisis de validez

Demostración de la validez de contenido

Demostración de la validez de criterio

Demostración de la validez de constructo (haciendo uso del procedimiento del Análisis factorial)

Al realizar un análisis factorial debe tenerse en cuenta, considerando los criterios de (Hair et al., 1 999), los elementos siguientes:

1 - La comprobación de los supuestos de este:

- Tamaño muestral

- Medida de suficiencia de muestreo (MSA)

- Prueba de esfericidad de Bartlett: Es una estadística que se utiliza para estudiar la presencia de correlaciones entre las variables, contrastándose dos hipótesis:

Ho: Las variables no están correlacionadas en la población, la matriz de correlaciones es una matriz identidad.

H1: Existe correlación entre las variables.

Para que el análisis factorial sea apropiado debe rechazarse la hipótesis nula.

- Medida de adecuación de la muestra de Kaiser- Meyer-Olkin(KMO): 
Todo lo que aparece abordado en textos de estadística y se puede obtener haciendo uso de paquetes de programas como el SPSS, el Statgraphics y/o Microsoft Excel.

\section{Análisis de los Resultados.}

Es necesario precisar que el instrumento (encuesta) aplicado tuvo dos formas de medición, una primera con 20 items relacionados con la calidad de vida de los ciudadanos, diagnosticando la situación real de la zona objeto de estudio y una segunda con 12 items relacionados con la adaptabilidad de la zona al desarrollo turístico como justificación y antecedente al proyecto que se propuso.

El análisis estadístico aplicado para demostrar la fiabilidad y la validez del instrumento se realizó por separado en función de los ítems estudiados.

Los resultados de la encuesta muestran que los mayores problemas están asociados al deterioro de las fachadas de las viviendas, no se perciben programas de reciclaje de desechos sólidos ni responsabilidad medioambiental por parte del gobierno y de las industrias enclavadas en la zona, escasa oferta gastronómica, alto grado de indisciplinas sociales y la no atención de la Policía Nacional Revolucionaria (PNR) ante estos hechos delictivos. También se declaran otros factores de impacto menos negativo como la no habilitación de cestos de basura, insuficiente limpieza e higiene, bajo nivel de conservación de los sitios con valores históricos y patrimoniales y escasa iluminación de las calles.

\section{Gráfico No. 1: Diagnóstico de la zona objeto de estudio. Incidencias negativas y menos negativas.}

\section{Frecuencia(-,--)}

INDISCIPLINAS SOCIALES. (VANDALISMO, ROBOS, ATENCIÓN A LA POBLACIÓN POR PARTE DE LA PNR ILUMINACIÓN DE LAS CALLES OFERTA DESARROLLADA POR COMERCIO Y PROMOCIÓN INDUSTRIAL Y GUBERNAMENTAL CON PROGRAMAS DE RECICLAJE DE DESECHOS SÓLIDOS NIVEL DE CONSERVACIÓN DE LOS SITIOS CON VALORES LIMPIEZA E HIGIENE EN ÁREAS COMUNES CESTOS HABILITADOS. FALLADA DE VIVIENDAS

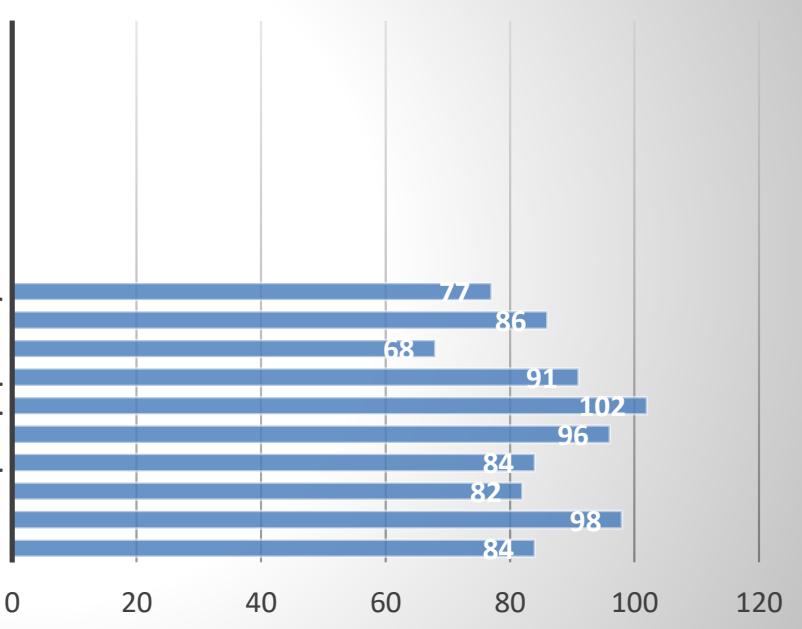

Fuente: Elaboración propia. 
Análisis de fiabilidad: Se utilizó el Alpha de Cronbach que debe ser mayor que 0.7 para considerarse aceptable. Los resultados muestran un coeficiente Alpha de Cronbach igual a 0,776 para N: 20. (Ver Anexo 6)

Tabla No. 1: Estadístico de fiabilidad del instrumento diseñado.

\begin{tabular}{|l|l|}
\hline $\begin{array}{l}\text { Alfa de } \\
\text { Cronbach }^{\mathrm{a}}\end{array}$ & $\begin{array}{l}\text { N de de } \\
\text { elementos }\end{array}$ \\
\hline .776 & 20 \\
\hline
\end{tabular}

Fuente: SPSS versión 20

Sobre la adaptabilidad de la zona al desarrollo turístico los encuestados señalan como atractivos de posible aprovechamiento la vida moderna, la religión, la arquitectura y las manifestaciones artísticas. Se asumen como beneficios la preservación del patrimonio, la higiene medioambiental, generación de empleos, diversificación de ofertas en la localidad y la integración de esta zona con otras del Centro Histórico; los problemas medioambientales, la pérdida de valores patrimoniales y los actos delictivos (droga, prostitución, robo, etc.) se declaran como factores de incidencia negativa del desarrollo del turismo en esa zona. Lo explicado anteriormente se muestra en el Gráfico No. 2:

Gráfico No. 2: Justificación y antecedentes al proyecto. Adaptabilidad al desarrollo turístico.

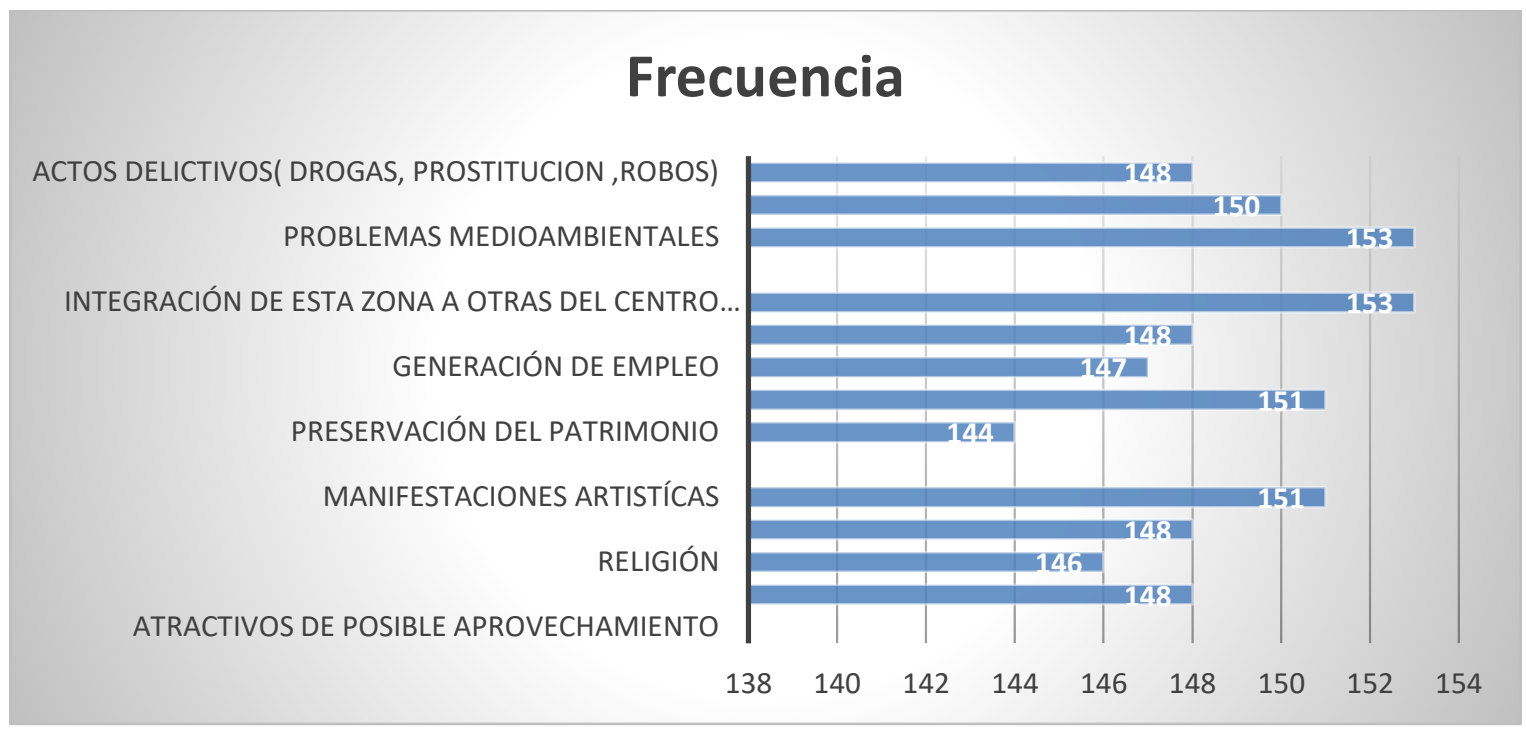

Fuente: Elaboración propia. 
Análisis de fiabilidad: Se utilizó el Alpha de Cronbach que debe ser mayor que 0.7 para considerarse aceptable. Los resultados muestran un coeficiente Alpha de Cronbach igual a 0,783 para N: 12 considerándose aceptable. (Ver Anexo 7)

Tabla No. 2: Estadístico de fiabilidad del instrumento diseñado.

\begin{tabular}{|l|l|}
\hline $\begin{array}{l}\text { Alfa de } \\
\text { Cronbach }^{\mathrm{a}}\end{array}$ & $\begin{array}{l}\mathrm{N} \\
\text { elementos }\end{array}$ \\
\hline .783 & 12 \\
\hline
\end{tabular}

Fuente: SPSS versión 20

De los análisis estadísticos anteriores resulta que el instrumento diseñado posee buena fiabilidad y validez.

Una vez aplicado el instrumento diseñado y encuestado a los pobladores de la zona objeto de estudio, estos demuestran la aceptación, en gran medida, de la incorporación de acciones que contribuyan a mejorar sus condiciones de vida y aseguran que estas mejoras, vinculadas con el turismo en esa zona, brindan beneficios sustanciales y equitativos en toda la comunidad.

En la siguiente figura se muestran las fases $\mathrm{y}$ actividades definidas para este tipo de proyecto.

Figura No. 2: Fases y actividades definidas para el Proyecto Comunitario (turísticocultural): Boulevard Maroya.

Fase 1: Conceptualización/
Inicio
- 1- Análisis de la situación
problémica.
- 2- Establecimiento de la
finalidad del proyecto.
- 3- Establecimiento del
Objetivo General.
-4- Identificación de las
partes interesadas.
-5- Análisis de las
necesidades de los
clientes.
-6- Análisis previo del
alcance del proyecto.
- 7- Estudio de viabilidad
inicial.

$\frac{\text { Fase 2: Diseño de }}{\text { configuración/ }}$
Planificación
- 1- Descripción detallada
del proyecto.
-2- Diseño operativo.
- 3- Gestión de riesgo.
- 4- Formación del equipo
del proyecto.
- 5- Certificación del
proyecto.
- 6- Elaboración del
manual de dirección del
proyecto.
- 7- Definición de los
subsistemas del
proyecto.
- 8- Estudio de
prefactibilidad.
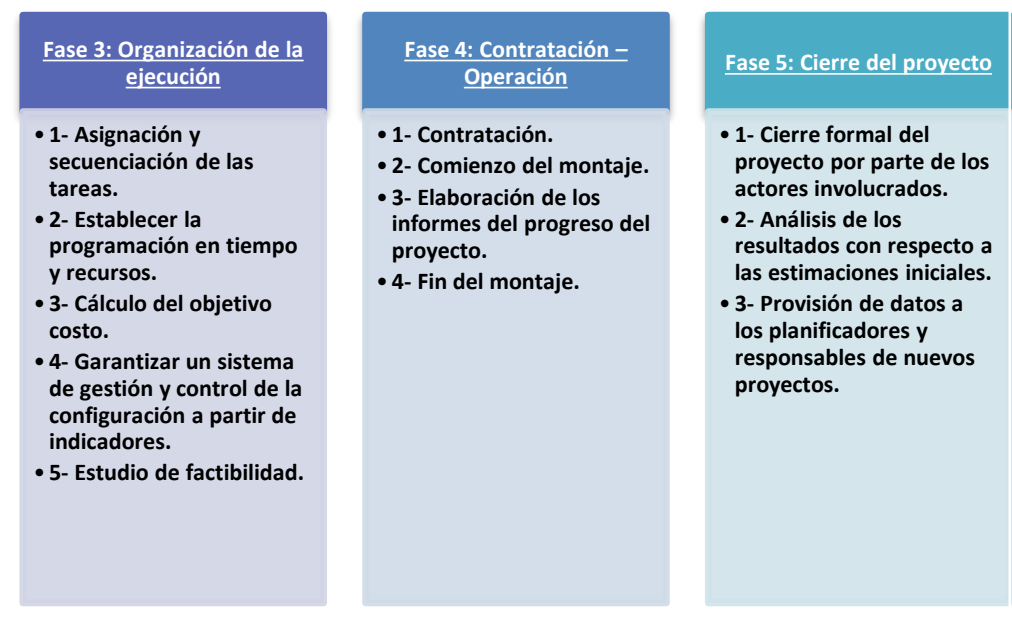

Fuente: Elaboración propia 


\section{Conclusiones.}

- La argumentación teórica y metodológica realizada, antecedida por la revisión bibliográfica permitió analizar diferentes conceptualizaciones acerca de la Dirección Integrada de Proyectos, los Proyectos Turísticos y el Turismo Cultural, así como la relación existente entre ellos.

- El estudio del estado del arte de los procedimientos existentes, posibilitó la creación del procedimiento para los Proyectos Comunitarios (TurísticosCulturales).

- Los métodos empleados permitieron diagnosticar la situación real existente en la zona objeto de estudio, así como analizar la adaptabilidad de la misma al desarrollo turístico.

- El análisis estadístico realizado demostró la fiabilidad y la validez de criterio, constructo y contenido del instrumento aplicado.

- Los resultados alcanzados, las bases diseñadas y la integración de los diferentes componentes, para el proyecto comunitario, constituyen una herramienta de vital importancia para insertar la zona objeto de estudio al desarrollo turístico del Centro Histórico de la ciudad.

\section{Referencias bibliográficas.}

Cleland, D \& King. W (1983) Systems Analysis and Project Management. Cited: 20 de noviembre de 2015.Available from: https://www.amazon.com/Systems-Analysis-ProjectManagement-Cleland/dp/007066224X

Fajardo, D. y Manresa, M., Metodología para la Gestión de Proyectos Turísticos. Revista TURyDES, Turismo y Desarrollo Local, junio 2012.

Gutiérrez y de Vara (2008.) Análisis y Diseño de Experimentos. Cited: 28 de septiembre de 2015. Available

from: https://www.researchgate.net/publication/44401609_Analisis_y_Diseno_de_Experimentos

Hair, J. F., Anderson, R. E., Tatham, R. L. \& Black, W. C. (1999). Análisis multivariado, Madrid, Prentice Hall.

Heredia, Rafael de. La Dirección Integrada de Proyecto (DIP) / Rafael de Heredia. -Madrid: Alianza, 1995 
PMBOK (2013). Project Management Book of Nnowledge (PMBOK) Fifth Edition Cited: 2 de enero de 2016. Available from:https://www.pmi.org/pmbok-guidestandards/foundational/pmbok

Zamora, T., Gestión y Evaluación de Proyectos Turísticos. Libro de texto docente. Universidad de La Habana, Manuscrito presentado para publicación. 2009 


\section{Para citar el artículo indexado.}

Sarduy M., Velastegui E. Bran. A., Gaibor A. \& Pazmiño M. (2018). La dirección integrada de proyectos aplicada a los proyectos comunitarios. Revista electrónica Ciencia Digital 2(3), 160-172. Recuperado desde: http://cienciadigital.org/revistacienciadigital2/index.php/CienciaDigital/article/view/144/12 $\underline{9}$

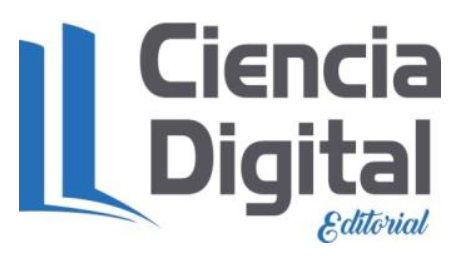

El artículo que se publica es de exclusiva responsabilidad de los autores y no necesariamente reflejan el pensamiento de la Revista Ciencia Digital.

El articulo queda en propiedad de la revista y, por tanto, su publicación parcial y/o total en otro medio tiene que ser autorizado por el director de la Revista Ciencia Digital.
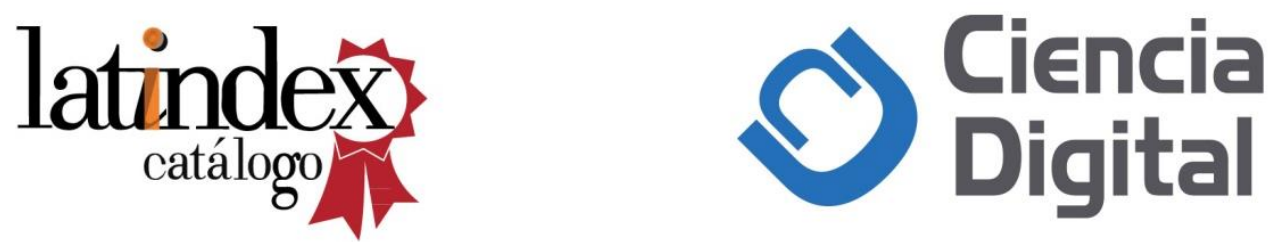